\title{
Adding Sensitivity to the MLBF Doppler Centroid Estimator
}

\author{
Ian G. Cumming, Member, IEEE, and Shu Li
}

\begin{abstract}
The multilook beat frequency (MLBF) algorithm is the Doppler centroid estimator most commonly used in practice to solve the Doppler ambiguity. However, it still makes errors, notably in medium- or low-contrast scenes. In this paper, we present two ways in which the estimation sensitivity of the MLBF algorithm can be improved. First, we give a more thorough frequencydomain explanation of how the MLBF algorithm works and explain how cross beating and range migration cause estimation difficulties. The first improvement to the algorithm replaces the fast Fourier transform (FFT)-based beat frequency estimator with a more accurate one that uses phase increments. It avoids the FFT limitations of resolution and quantization, especially when the signal is discontinuous in one range cell due to range cell migration or burst mode operation (ScanSAR). A second improvement uses range cell migration correction to straighten the target trajectories before the beat frequency estimator is applied. This has the effect of narrowing the bandwidth of the beat signal and reducing the effect of cross beating. Finally, experiments with RADARSAT-1 data are used to illustrate the improved estimation accuracy of the modified algorithm.
\end{abstract}

Index Terms-Burst mode SAR, Doppler ambiguity resolution, Doppler centroid estimation, frequency estimation, multilook beat frequency (MLBF), range cell migration correction (RCMC), SAR antenna pointing angle, ScanSAR, synthetic aperture radar (SAR).

\section{INTRODUCTION}

I N PRECISION synthetic aperture radar (SAR) processing, accurate estimation of the Doppler centroid frequency is essential for good image quality. This is especially true in interferometric applications where aligned frequencies are needed, and in ScanSAR processing where radiometric correction depends strongly on the Doppler frequency.

In SAR systems, the azimuth data are acquired in a sampled fashion, with the sampling rate given by the pulse repetition frequency (PRF). Because of the spectral repetition of the sampled data, the Doppler centroid estimate is usually observed in two parts: the baseband Doppler centroid (expressed as a fraction of the PRF) and the Doppler ambiguity (expressed as an integer multiple of the PRF). For example, if the absolute Doppler centroid is $5200 \mathrm{~Hz}$ and the PRF is $1000 \mathrm{~Hz}$, the baseband centroid is 0.2 PRFs, and the Doppler ambiguity is +5 .

Manuscript received December 23, 2005; revised May 12, 2006. This work was supported in part by the Natural Sciences and Engineering Research Council and in part by MacDonald Dettwiler and Associates.

The authors are with the Department of Electrical and Computer Engineering, The University of British Columbia, Vancouver, BC V6T 1Z4, Canada (e-mail: ianc@ece.ubc.ca; shul@ece.ubc.ca).

Color versions of one or more of the figures in this paper are available online at http://ieeexplore.ieee.org.

Digital Object Identifier 10.1109/TGRS.2006.887010
For observability and accuracy reasons, separate estimators are needed for each of these two parts.

In the baseband Doppler centroid estimation, several algorithms, such as the "Spectral fit" and average crosscorrelation (ACCC) methods, give reliable estimates in most cases - usually better than $1 \%$ of the PRF [1]. In solving for the Doppler ambiguity number, several algorithms have been used, such as the look displacement [2], multiple PRF [3], wavelength diversity (WDA) [4], multilook cross correlation (MLCC) [5], and multilook beat frequency (MLBF) algorithms [5]. However, the accuracy and robustness of the Doppler ambiguity number estimate still need improvement to satisfy current high-quality SAR processing requirements. ${ }^{1}$

The MLBF algorithm proposed in 1996 is the most reliable of the current ways of estimating the Doppler ambiguity of SAR signals. The method is based on extracting two looks from the data in the range-frequency domain, beating (conjugate multiplying) the two looks together, then estimating the center frequency of the beat signal. Extracting two looks emulates the signal from two radars with center frequencies separated by approximately half the range bandwidth, and beating the signals extracts their phase difference. The center frequency of the beat signal is proportional to the absolute Doppler centroid, and unwrapping is not needed because of the closeness of the center frequencies of the two looks.

Estimating the absolute Doppler centroid from the center frequency works well in high-contrast scenes but does not always get the correct ambiguity number in medium- or lowcontrast scenes. The WDA and MLCC algorithms work better in low-contrast scenes but suffer from the calibration problem of an offset frequency arising from the antenna characteristics [6]. In this paper, we analyze the phase history of the beat signal in the frequency domain then propose two methods to improve the performance of the existing MLBF algorithm.

\section{PRINCIPLES OF THE MLBF Algorithm}

The MLBF algorithm takes advantage of the differences in azimuth phase history between two range looks to estimate the Doppler centroid. The azimuth signals of the two range looks are beat together, and the frequency of the beat signal is estimated. The properties of the beat signal are explained in the next two sections.

\footnotetext{
${ }^{1}$ When yaw steering is practiced and the attitude control is precise, there is not so much need for ambiguity estimation. However, yaw steering can be turned OFF for specific experiments, and there is a tendency for MacDonald Dettwiler satellite's attitude control system to degrade with time. In these cases, the accuracy of the Doppler ambiguity resolver (DAR) algorithms is a concern.
} 


\section{A. Phase Relationship Between the Time and Frequency Domains}

In order to explain the principle of the MLBF algorithm, we consider the signal model for a single point target. As the algorithm operates on range-compressed data, the compressed signal in the range-frequency domain $f_{\tau}$ can be expressed as

$$
S_{\mathrm{RC}}\left(f_{\tau}, \eta\right)=W_{r}\left(f_{\tau}\right) w_{a}\left(\eta-\eta_{c}\right) \exp \left\{-j \frac{4 \pi\left(f_{0}+f_{\tau}\right) R(\eta)}{c}\right\}
$$

where $f_{0}$ is the radar center frequency, $R(\eta)$ is the slant range versus time $\eta, c$ is the propagation speed of the signal, $W_{r}\left(f_{\tau}\right)$ is the envelope of the range-frequency spectrum, and $w_{a}\left(\eta-\eta_{c}\right)$ is the azimuth envelope with respect to the beam center crossing time $\eta_{c}$.

As shown in (1), the phase of a range-compressed signal in the range-frequency domain consists of a constant term $-4 \pi f_{0} R(\eta) / c$ and a term $-4 \pi f_{\tau} R(\eta) / c$ that is linear with respect to the range frequency. The second term is important to the MLBF algorithm. It represents a phase ramp in the range-frequency domain, which has a characteristic slope $-4 \pi R(\eta) / c$. Since the slant range $R(\eta)$ varies in azimuth due to the range migration, the slope of the phase ramp changes with time (along the azimuth direction).

The Fourier transform (FT) can be used to observe the relationship between the signal in the range time and the rangefrequency domains. Using a range IFT on (1), the rangecompressed signal can be expressed in the time domain as [6]

$$
\begin{aligned}
s_{\mathrm{rc}}(\tau, \eta) & =\operatorname{IFT}_{r}\left\{S_{\mathrm{RC}}\left(f_{\tau}, \eta\right)\right\} \\
& =p_{r}(\tau-2 R(\eta) / c) w_{a}\left(\eta-\eta_{c}\right) \exp \left\{-j 4 \pi f_{0} R(\eta) / c\right\}
\end{aligned}
$$

where the linear phase ramp in (1) has been converted into a range shift of $2 R(\eta) / c$ seconds.

To observe the phase properties of the signal, it is convenient to consider a range-compressed pulse whose peak occurs at range time $\tau=0$. The value of the time-domain signal at its peak can be found using the inverse FT of the frequencydomain signal (1)

$$
\begin{aligned}
s_{\mathrm{rc}}(0, \eta) & =\left.\int_{f_{\tau} \min }^{f_{\tau} \max } S_{\mathrm{RC}}\left(f_{\tau}, \eta\right) \exp \left\{j 2 \pi f_{\tau} \tau\right\} d f_{\tau}\right|_{\tau=0} \\
& =\int_{f_{\tau} \min }^{f_{\tau} \max } S_{\mathrm{RC}}\left(f_{\tau}, \eta\right) d f_{\tau} .
\end{aligned}
$$

Because of the equality in (3), the phases of the left- and right-hand sides must be equal. Therefore, we can derive the phase of the range-compressed pulse from the integral in the frequency domain

$$
\arg \left\{s_{\mathrm{rc}}(0, \eta)\right\}=\arg \left\{\int_{f_{\tau} \min }^{f_{\tau} \max } S_{\mathrm{RC}}\left(f_{\tau}, \eta\right) d f_{\tau}\right\} .
$$

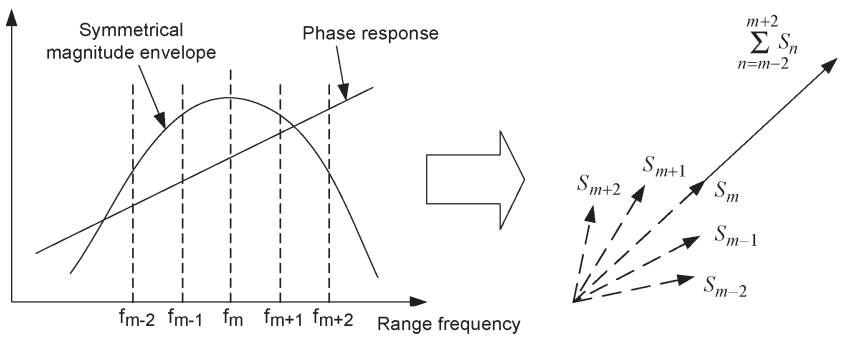

(a)

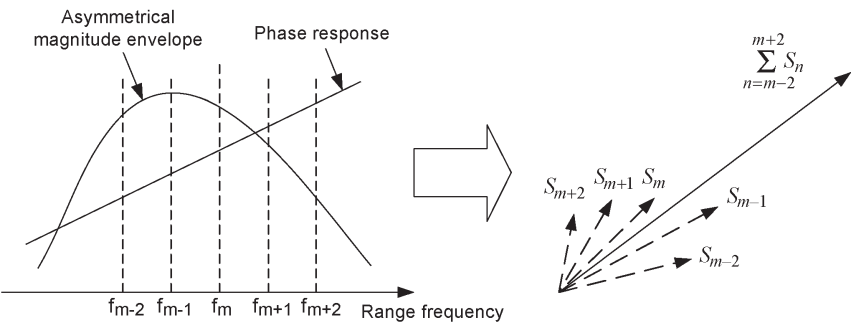

(b)

Fig. 1. Illustrating how asymmetrical range looks shift the observed "central frequency" in the range spectrum. (a) Phase calculation from symmetric spectrum. (b) Phase calculation from asymmetric spectrum.

This integral relationship is very important when analyzing the phase properties of the two range looks used in the MLBF algorithm. It also shows the importance of symmetrical look extraction - if the look magnitudes are symmetrical, the phase of the compressed pulse equals the phase at the center of the spectrum, which simplifies the calibration of the MLBF algorithm.

To better illustrate how the phase is computed, we introduce a variable called "central frequency" $f_{\tau_{c}}$. The central frequency is defined as the frequency at which the phase of the range spectrum (1) equals the phase of the integrated spectrum on the right hand side of (3) and thus equals the phase of the compressed pulse. This definition can be expressed as

$$
\begin{aligned}
\arg \left\{S_{\mathrm{RC}}\left(f_{\tau_{c}}, \eta\right)\right\} & =\arg \left\{\int_{f_{\tau} \min }^{f_{\tau} \max } S_{\mathrm{RC}}\left(f_{\tau}, \eta\right) d f_{\tau}\right\} \\
& =\arg \left\{s_{\mathrm{rc}}(0, \eta)\right\} .
\end{aligned}
$$

Given that the target phase is linear in range frequency, the magnitude of the frequency response must have a symmetrical shape to guarantee that the central frequency is located at the center of the spectrum. Fig. 1 illustrates the importance of the symmetrical magnitude using a simple discrete case. When the spectrum has a symmetrical shape, as shown in Fig. 1(a), the summation of the five frequency components (represented by the five dashed vectors) has the same phase as the central term.

In contrast, when the spectrum has an asymmetrical shape, as shown in Fig. 1(b), the phase of the summation has an offset from the phase of the center of the spectrum. It is shown that only when the spectrum has a symmetrical magnitude envelope can the central frequency $f_{\tau_{c}}$ be obtained directly from the frequency of the central term $f_{\text {center }}$. Otherwise, the 


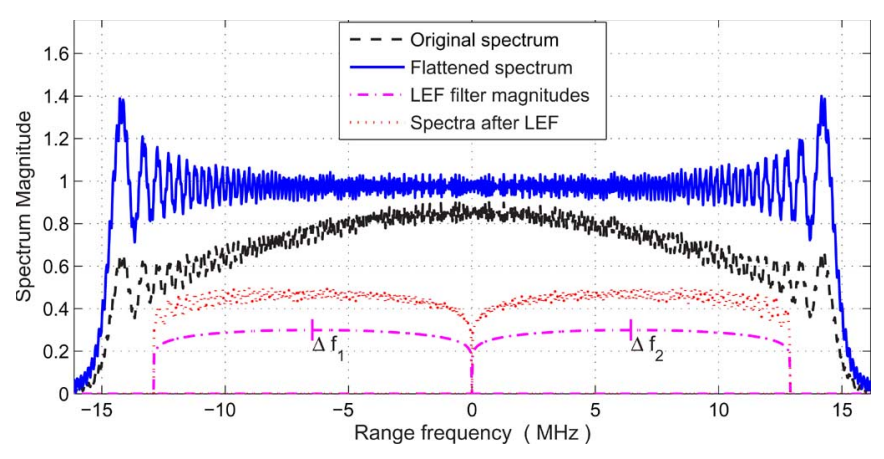

Fig. 2. Illustration of symmetrical look extraction from the range spectrum.

two frequencies are not equal $\left(f_{\tau_{c}} \neq f_{\text {center }}\right)$, and a calibration factor is needed to compensate the difference. ${ }^{2}$

\section{B. Range Look Extraction}

In the MLBF algorithm, we extract two looks from the rangefrequency domain. In order to obtain the symmetry discussed above, it is expedient to flatten the range spectrum then apply symmetrical look-extraction filters. Since the uneven edges of the range spectrum may affect the symmetry of the look spectra, the look-extraction windows are tapered to minimize the edge effects.

Fig. 2 illustrates the procedure of extracting two range looks using symmetrical windows. The original range spectrum, the spectrum after flattening, the look-extraction filter shapes, and the spectra of the extracted looks are shown. The variables $\Delta f_{1}=-6.47 \mathrm{MHz}$ and $\Delta f_{2}=+6.47 \mathrm{MHz}$ are the frequency offsets of each look. As we assume that the range spectrum has been demodulated to baseband (zero center frequency), they are the actual center frequencies of each look. As the spectra of the extracted looks are symmetrical, $\Delta f_{1}$ and $\Delta f_{2}$ also correspond to the "central frequency" $f_{\tau_{c}}$ of the two looks.

After extraction, the range looks have a nonzero center frequency, i.e., they are not at baseband. As illustrated in [6, eq. (3.33) and Fig. 3.8], nonbaseband targets have a linear phase ramp through the peak of the pulse. If the central frequency of the range spectrum is $\Delta f$, the phase ramp equals $2 \pi \Delta f\left(\tau-\tau_{0}\right)$. According to this theory, the extracted looks can be expressed as follows in the range time domain:

$$
\begin{aligned}
s_{r_{1} \_\mathrm{nb}}(\tau, \eta)= & \operatorname{IFFT}_{r}\left\{S_{\mathrm{RCF}}\left(f_{\tau}, \eta\right) W_{1}\left(f_{\tau}\right)\right\} \\
= & A_{1} p_{r}(\tau-2 R(\eta) / c) w_{a}\left(\eta-\eta_{c}\right) \\
& \times \exp \left\{-j 4 \pi f_{0} R(\eta) / c\right\} \\
& \times \exp \left\{-j 2 \pi \Delta f_{1}(\tau-2 R(\eta) / c)\right\}
\end{aligned}
$$

and

$$
\begin{aligned}
s_{r_{2} \_\mathrm{nb}}(\tau, \eta)= & \operatorname{IFFT}_{r}\left\{S_{\mathrm{RCF}}\left(f_{\tau}, \eta\right) W_{2}\left(f_{\tau}\right)\right\} \\
= & A_{1} p_{r}(\tau-2 R(\eta) / c) w_{a}\left(\eta-\eta_{c}\right) \\
& \times \exp \left\{-j 4 \pi f_{0} R(\eta) / c\right\} \\
& \times \exp \left\{-j 2 \pi \Delta f_{2}(\tau-2 R(\eta) / c)\right\}
\end{aligned}
$$

${ }^{2}$ In the results in this paper, the look extraction has made the spectrum symmetrical; therefore, a calibration factor is not needed. where $s_{r_{1} \_\mathrm{nb}}(\tau, \eta)$ and $s_{r_{2} \_ \text {nb }}(\tau, \eta)$ are the signals of the range looks extracted from nonbaseband spectra, $S_{\mathrm{RCF}}\left(f_{\tau}, \eta\right)$ is the flattened version of the range-compressed spectrum (1), and $W_{1}\left(f_{\tau}\right)$ and $W_{2}\left(f_{\tau}\right)$ are the magnitudes of the range lookextraction filters.

The second phase terms in (6) and (7) show that the rangecompressed pulses contain phase ramps over the main lobe $2 \pi \Delta f_{1} \tau$ and $2 \pi \Delta f_{2} \tau$, with a different slope for each look. However, as we are interested in the phase changes in the azimuth direction, these range direction phases are distracting. In order for the azimuth phases of the two looks to be easily compared, the range center frequencies of the two looks can be moved to the same frequency. For conceptual simplicity, the new center frequencies will be moved to zero (baseband), where the phases over the main lobe of the compressed pulses are flat.

This bandshifting is done after the look-extraction step. As the shift in the frequency domain corresponds to a modulation in the time domain, the signal of two extracted looks after the spectrum shift can be expressed in the time domain as

$$
\begin{aligned}
\text { Look 1: } 1: s_{r_{1}}(\tau, \eta)= & \operatorname{IFFT}_{r}\left\{S_{\mathrm{RCF}}\left(f_{\tau}+\Delta f_{1}, \eta\right)\right. \\
& \left.\times W_{1}\left(f_{\tau}+\Delta f_{1}\right)\right\} \\
= & A_{1} p_{r}(\tau-2 R(\eta) / c) w_{a}\left(\eta-\eta_{c}\right) \\
& \times \exp \left\{-j 4 \pi\left(f_{0}+\Delta f_{1}\right) R(\eta) / c\right\}
\end{aligned}
$$

and

Look $2: \quad s_{r_{2}}(\tau, \eta)=\operatorname{IFFT}_{r}\left\{S_{\mathrm{RCF}}\left(f_{\tau}+\Delta f_{2}, \eta\right)\right.$

$$
\begin{gathered}
\left.\times W_{2}\left(f_{\tau}+\Delta f_{2}\right)\right\} \\
=A_{1} p_{r}(\tau-2 R(\eta) / c) w_{a}\left(\eta-\eta_{c}\right) \\
\times \exp \left\{-j 4 \pi\left(f_{0}+\Delta f_{2}\right) R(\eta) / c\right\}
\end{gathered}
$$

where $s_{r_{1}}(\tau, \eta)$ and $s_{r_{2}}(\tau, \eta)$ are the signals of the range looks extracted from the baseband spectra. After this bandshifting, the $\tau$ dependence of the phase is removed, and the extracted looks are appropriately conditioned to generate the beat signal in azimuth.

\section{Properties of the Beat Signal}

After the bandshifting, the beat signal can be derived from the two baseband looks (8) and (9) by multiplying one look with the conjugate of the other ${ }^{3}$

$$
\begin{aligned}
s_{\text {beat }}(\tau, \eta)= & s_{r_{1}}^{*}(\tau, \eta) s_{r_{2}}(\tau, \eta) \\
= & \left|A_{1} w_{a}\left(\eta-\eta_{c}\right)\right|^{2} \\
& \times \exp \left\{-j 4 \pi \Delta f_{r} R(\eta) / c\right\}
\end{aligned}
$$

\footnotetext{
${ }^{3}$ The range envelope and migration factor $\left[p_{r}(\tau-2 R(\eta) / c)\right]$ has been removed from this equation, and subsequent equations for simplicity. If the RCM is very small, then the target lies entirely within one range cell and so does the beat signal. If the RCM extends over several cells, the beat signal will also extend over the same number of cells, and the exposure in any one cell is correspondingly smaller. However, when the signal increments are averaged over range (see Section IV-C), the result is very similar to the case where there is negligible RCM.
} 
where $\Delta f_{r}=\Delta f_{2}-\Delta f_{1}$ is the frequency difference of the two range looks before the bandshifting.

It can be seen from (10) that the phase of the beat signal varies with azimuth time because of the change of the slant range function $R(\eta)$. If we expand $R(\eta)$ about the central illuminated time $\eta_{c}$ and ignore the small higher order components, the range between the target and the radar can be approximated by the parabola [6]

$$
R(\eta) \approx R\left(\eta_{c}\right)-V_{r} \sin \theta_{r, c}\left(\eta-\eta_{c}\right)+\frac{1}{2} \frac{V_{r}^{2} \cos ^{2} \theta_{r, c}}{R\left(\eta_{c}\right)}\left(\eta-\eta_{c}\right)^{2}
$$

where $V_{r}$ is the "effective radar velocity" and $\theta_{r, c}$ is the beam squint angle measured in the slant range plane [6]. Note that $R(\eta)$ of (11) has linear and quadratic components. The linear component imparts a pure sine wave to the beat signal, whose frequency is found by differentiating the phase term of (10)

$$
\begin{aligned}
f_{\text {beat }} & =-\frac{2 \Delta f_{r}}{c} \frac{d R(\eta)}{d \eta} \\
& =\frac{2 \Delta f_{r}}{c} V_{r} \sin \theta_{r, c} \\
& =-\frac{\Delta f_{r}}{f_{0}} f_{\eta_{c}}
\end{aligned}
$$

where $f_{\eta_{c}}=-2 V_{r} \sin \theta_{r, c} / \lambda$ is the Doppler centroid frequency.

Similarly, the quadratic component of (11) imparts a nonzero bandwidth to the beat signal given by

$$
\begin{aligned}
\mathrm{BW}_{\text {beat }} & =\frac{2 \Delta f_{r}}{c} \frac{d^{2} R(\eta)}{d \eta^{2}} T_{a} \\
& =\frac{\Delta f_{r} V_{r}^{2} \cos ^{2} \theta_{r, c}}{c R\left(\eta_{c}\right)} T_{a} \\
& =\frac{\Delta f_{r}}{f_{0}} \mathrm{BW}_{\text {azimuth }}
\end{aligned}
$$

where $\mathrm{BW}_{\text {azimuth }}=2 V_{r}^{2} \cos ^{2} \theta_{r, c} T_{a} /\left[\lambda R\left(\eta_{c}\right)\right]$ is the azimuth bandwidth of the received signal, $T_{a}$ is the effective exposure time of a target, and $\lambda=c / f_{0}$ is the radar wavelength. The quadratic component does not change the average frequency of the beat signal, which is governed by the average range cell migration (RCM) component (i.e., the linear component).

Both the center frequency $f_{\text {beat }}$ and the bandwidth $\mathrm{BW}_{\text {beat }}$ of the beat signal are quite small compared to the system PRF. Taking the RADARSAT-1 Vancouver scene as an example, where $f_{\eta_{c}} \approx-5900 \mathrm{~Hz}, \Delta f_{r}=14 \times 10^{6} \mathrm{~Hz}, f_{0}=5.3 \times$ $10^{9} \mathrm{~Hz}$, and BW $\mathrm{Bz}_{\text {azimuth }} \approx 950 \mathrm{~Hz}$, it is found that $f_{\text {beat }}$ is about $16 \mathrm{~Hz}$, and $\mathrm{BW}_{\text {beat }}$ is about $2.5 \mathrm{~Hz}$. The small value of $f_{\text {beat }}$ compared to the PRF of $1275 \mathrm{~Hz}$ means that the estimated beat frequency is well away from the aliasing limits of $\pm \mathrm{PRF} / 2$; therefore, there is no ambiguity present in the estimate of the beat frequency. Also, the nonzero bandwidth of the beat signal does not cause a significant frequency estimation problem as it is in the order of magnitude of the ambiguity error limits.
The error limits are given by the beat frequency deviations caused by Doppler centroid estimation errors of $\pm \mathrm{PRF} / 2$, which give beat frequency error limits of $\pm 1.7 \mathrm{~Hz}$ in this case. The bandwidth is larger for L-band satellites, but the ambiguity error limits are correspondingly larger.

At this point, the importance of the magnitude spectrum being symmetrical is recognized. If the looks are not symmetrical, the average frequency calculated from the beat spectrum will not equal (12), and a different look separation $\Delta f_{r}$ must be "calibrated."

\section{Summary}

In summary, the beat signal has an average frequency proportional to the absolute Doppler centroid frequency, which we are trying to estimate. The proportionality factor is the fractional separation of the range looks $\Delta f_{r} / f_{0}$. There is also a nonzero bandwidth in the beat signal, but it is relatively small. The narrow bandwidth justifies that the beat signal can be approximated by a single frequency with noise when only one target is present. The relative size of the noise depends on the image signal-to-noise ratio (SNR) and scene content, as well as the factors discussed in the next section.

\section{Distortion of the Beat Spectrum}

In this section, we discuss two phenomena that can introduce noise and distortion into the beat spectrum — cross beating and range migration.

\section{A. Cross Beating of Multiple Targets}

The MLBF concept outlined in Section II is based on a single target in each range cell, which leads to the derivation of the ideal beat signal (10). However, in practice, there is inevitably more than one target in each range cell. This leads to a cross-beating effect that distorts the beat signal [5]. The cross beating arises when Look 1 of one target beats with Look 2 of other targets. The beating between different targets (cross beating) gives rise to spurious frequency components in the beat spectrum. The resulting distortion of the beat spectrum depends on the number, strength, and distribution of the extra targets, ultimately giving it a noiselike appearance.

In order to understand the effect of the cross beating, we first analyze the case of two targets. Consider two targets $P$ and $Q$ that are in the same range cell but are separated in azimuth, with beam center crossing times $\eta_{P}$ and $\eta_{Q}$. Then, the signals of the two range looks $s_{1}(\eta)$ and $s_{2}(\eta)$ can be expressed as [5]

$$
\text { Look 1: } \begin{aligned}
s_{1}(\eta)= & s_{P_{1}}(\eta)+s_{Q_{1}}(\eta) \\
= & A_{P} w_{a}\left(\eta-\eta_{P}\right) \\
& \times \exp \left\{-j 4 \pi\left(f_{0}+\Delta f_{1}\right) R\left(\eta-\eta_{P}\right) / c\right\} \\
& +A_{Q} w_{a}\left(\eta-\eta_{Q}\right) \\
& \times \exp \left\{-j 4 \pi\left(f_{0}+\Delta f_{1}\right) R\left(\eta-\eta_{Q}\right) / c\right\}
\end{aligned}
$$


and

$$
\text { Look } 2: \quad \begin{aligned}
s_{2}(\eta)= & s_{P_{2}}(\eta)+s_{Q_{2}}(\eta) \\
= & A_{P} w_{a}\left(\eta-\eta_{P}\right) \\
& \times \exp \left\{-j 4 \pi\left(f_{0}+\Delta f_{2}\right) R\left(\eta-\eta_{P}\right) / c\right\} \\
& +A_{Q} w_{a}\left(\eta-\eta_{Q}\right) \\
& \times \exp \left\{-j 4 \pi\left(f_{0}+\Delta f_{2}\right) R\left(\eta-\eta_{Q}\right) / c\right\}
\end{aligned}
$$

where $A_{P}$ and $A_{Q}$ are the amplitudes of the targets after range compression and look extraction. Then, the beat signal becomes

$$
\begin{aligned}
s_{\text {beat }}(\eta)= & s_{1}^{*}(\eta) s_{2}(\eta) \\
= & s_{P_{1}}^{*}(\eta) s_{P_{2}}(\eta)+s_{Q_{1}}^{*}(\eta) s_{Q_{2}}(\eta) \\
& +s_{P_{1}}^{*}(\eta) s_{Q_{2}}(\eta)+s_{Q_{1}}^{*}(\eta) s_{P_{2}}(\eta) .
\end{aligned}
$$

In (16), the first term represents the beat signal generated by Target $P$ alone, and the second term represents the beat signal generated by Target $Q$ alone. These two "autobeat" terms provide the correct beat frequency, as in the case of a single target. However, the third and fourth terms represent the crossbeat signals generated by Target $P$ in one look beating with Target $Q$ in the other look.

The cross beating leads to extra frequencies in the beat signal spectrum. The third term of (16) can be expanded as

$$
\begin{aligned}
& s_{P_{1}}^{*}(\eta) s_{Q_{2}}(\eta)= A_{P}^{*} A_{Q} w_{a}\left(\eta-\eta_{P}\right) w_{a}\left(\eta-\eta_{Q}\right) \\
& \times \exp \left\{j 4 \pi \left[\left(f_{0}+\Delta f_{1}\right) R\left(\eta-\eta_{P}\right)\right.\right. \\
&\left.\left.-\left(f_{0}+\Delta f_{2}\right) R\left(\eta-\eta_{Q}\right)\right] / c\right\} .
\end{aligned}
$$

Using the expansion for $R(\eta)$ of (11), the frequency of this cross term is

$$
\begin{aligned}
f_{P^{*} Q} & =\left.\frac{2}{c} \frac{d\left[\left(f_{0}+\Delta f_{1}\right) R\left(\eta-\eta_{P}\right)-\left(f_{0}+\Delta f_{2}\right) R\left(\eta-\eta_{Q}\right)\right]}{d \eta}\right|_{\eta=\eta_{c}} \\
& =\frac{2}{c}\left(\Delta f_{2}-\Delta f_{1}\right) V_{r} \sin \theta_{r, c}+\frac{2}{c} \frac{V_{r}^{2} \cos ^{2} \theta_{r, c}}{R\left(\eta_{c}\right)} f_{0}\left(\eta_{P}-\eta_{Q}\right) \\
& =f_{\text {beat }}+K_{a} \Delta \eta
\end{aligned}
$$

where $f_{\text {beat }}$ is the single-target beat frequency defined in (12), $K_{a}=2 V_{r}^{2} \cos ^{2} \theta_{r, c} /(\lambda R)$ is the Doppler FM rate of the signal, and $\Delta \eta=\eta_{P}-\eta_{Q}$ is the separation of the two targets in azimuth time. Similarly, the cross frequency of the fourth term of (16) can be expressed as

$$
f_{P Q^{*}}=f_{\text {beat }}-K_{a} \Delta \eta
$$

Equations (18) and (19) represent the extra frequencies in the beat signal spectrum due to cross beating. Note that the offset $\pm K_{a} \Delta \eta$ of the cross-beat frequencies from the expected beat frequency depends on the Doppler FM rate and the target separation but does not depend on the look separation $\left(\Delta f_{2}-\Delta f_{1}\right)$. (a)

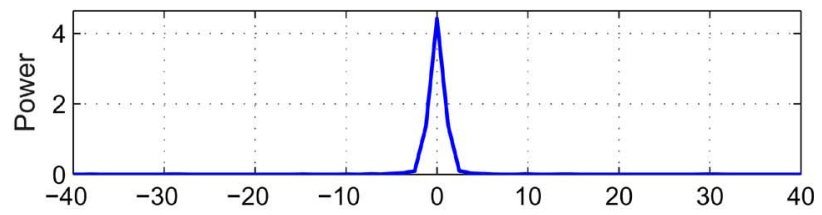

(b)

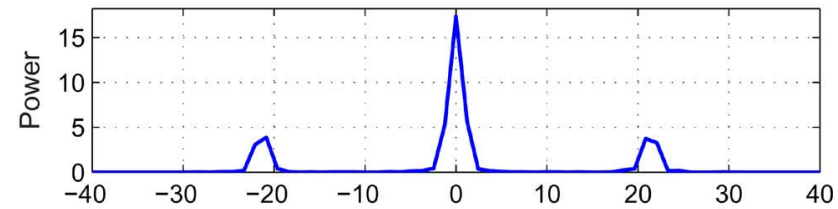

(c)

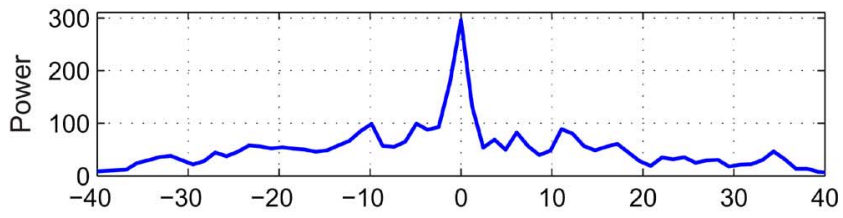

(d)

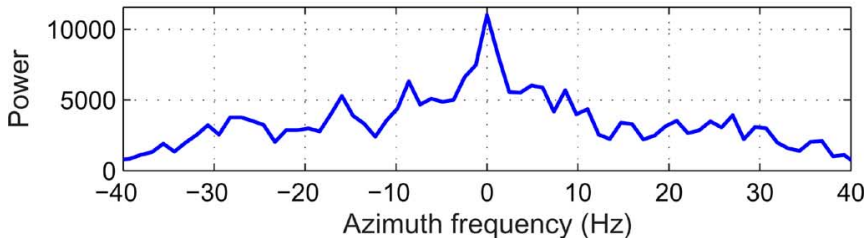

Fig. 3. Effect of cross beating on the beat spectrum (no linear RCM). (a) Beat spectrum of one target. (b) Beat spectrum with two targets. (c) Beat spectrum with 100 targets. (d) Beat spectrum with 1000 targets.

The amplitude of the cross-beating signals is usually less than the main beat signal, as one target is often smaller than the other target, and because the duration of the overlap of the two targets is less than the exposure time of one target. Thus, the beat spectrum is not distorted much if $\left|A_{P}\right| \gg\left|A_{Q}\right|$ and/or if the targets are well separated in azimuth. However, the effect of the cross beating on the spectrum becomes noticeable when $A_{Q}$ becomes close to $A_{P}$ in magnitude, and there is substantial overlap between the targets.

Fig. 3 uses a discrete-target simulation to illustrate the crossbeating effect that occurs when more than one target is present in the same range cell. ${ }^{4}$ Panel (a) shows the beat spectrum of single target in one range cell, and Panel (b) shows the beat spectrum of two overlapping targets. For simplicity, the Doppler centroid is assumed to be zero; therefore, there is no net range migration, and the expected beat frequency is zero.

Comparing the top two panels, it can be seen that the cross beating creates two extra peaks in the beat spectrum of Panel (b). The strengths of the two targets are the same, and the azimuth target separation is 15 pulses or $0.01193 \mathrm{~s}^{5}$ The exposure time of each target is 700 pulses or $0.55 \mathrm{~s}$, the PRF is $1257 \mathrm{~Hz}$, the FM rate is $-1790 \mathrm{~Hz} / \mathrm{s}$, and the parameters are taken from the RADARSAT-1 FINE mode. With these

\footnotetext{
${ }^{4}$ The horizontal axes in Figs. 3, 5, 6, 8, and 9 refer to the frequency of the beat signal, which is a scaled version of azimuth frequency.

${ }^{5}$ The target phase has no effect on the beat spectrum, as the beating subtracts the phases of the two looks.
} 


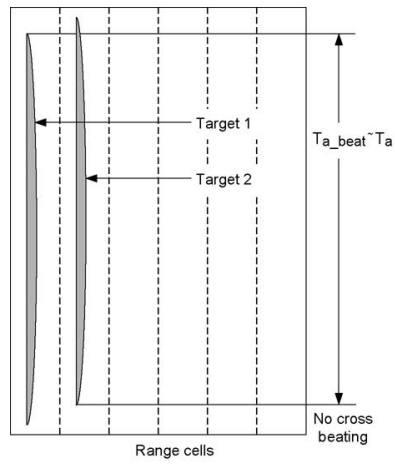

(a)

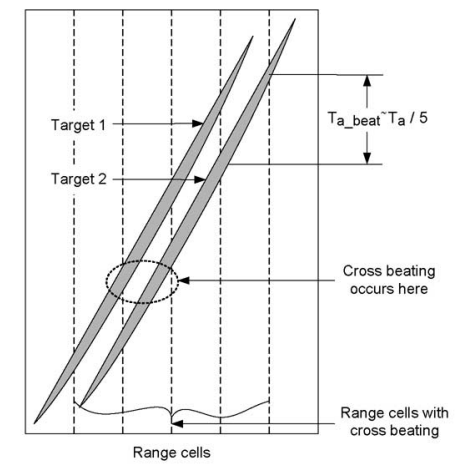

(b)
Fig. 4. Distribution of the energy of two targets in range-compressed data. (a) Target energy with no linear RCM. (b) Target energy with significant RCM.

parameters, the 15-cell azimuth separation gives a frequency shift of $\pm 21.4 \mathrm{~Hz}$ for the cross-beat components, which is in agreement with (18) and (19). Note that the strength of the cross-beat signal does not depend on the relative phase of the two targets, but the phase does, which has an effect on the multitarget results of Panel (c).

Extending the argument from two targets to multiple targets, it is clear that many more cross-beat components will be observed in the beat spectrum. This effect is illustrated in Panels (c) and (d). In these simulations, there are 100 [Panel (c)] or 1000 [Panel (d)] targets located randomly in a $30 \times 30$ sample image area. The target amplitudes are equal at one unit, and Gaussian noise is added with an rms value of unity. The relative target phases are random. The cross-beating components in the same range cell add coherently, which can result in spurious peaks and nulls in the beat spectrum.

In general, the cross beating increases with the density of the targets. When the density is too high, the beat frequency may not be detected within the ambiguity error limits. That is why it is more difficult to estimate the beat frequency when there are many targets with similar magnitude, as in homogeneous low-contrast scenes. More cross-beating examples are shown in Fig. 5 and in the RADARSAT examples of Section VI.

\section{B. Effect of Range Migration on the Beat Spectrum}

A significant amount of RCM is often present in satellite SAR data. The effect of RCM on target overlap is illustrated in Fig. 4, which compares the trajectories of two targets that have a small displacement in range and azimuth. Panel (a) shows the locus of target energy in the range-compressed data with no linear RCM, which can be the case when the beam is steered to zero Doppler. When the Doppler centroid is well away from zero, significant RCM can be present, as illustrated in Panel (b).

Two effects are noticed in Fig. 4. First, the exposure time of each target is reduced by the RCM, when observed within a single range cell. In this example, the exposure time within a range cell has been reduced to $T_{a} / 5$ by the five-cell RCM, where $T_{a}$ is the full exposure time of each target. As the beat signal is generated within each range cell, the reduced exposure time means that the resolution of the beat signal is broadened, which lowers the resolution of the estimation process. Second, while there is only one target in each range cell when there
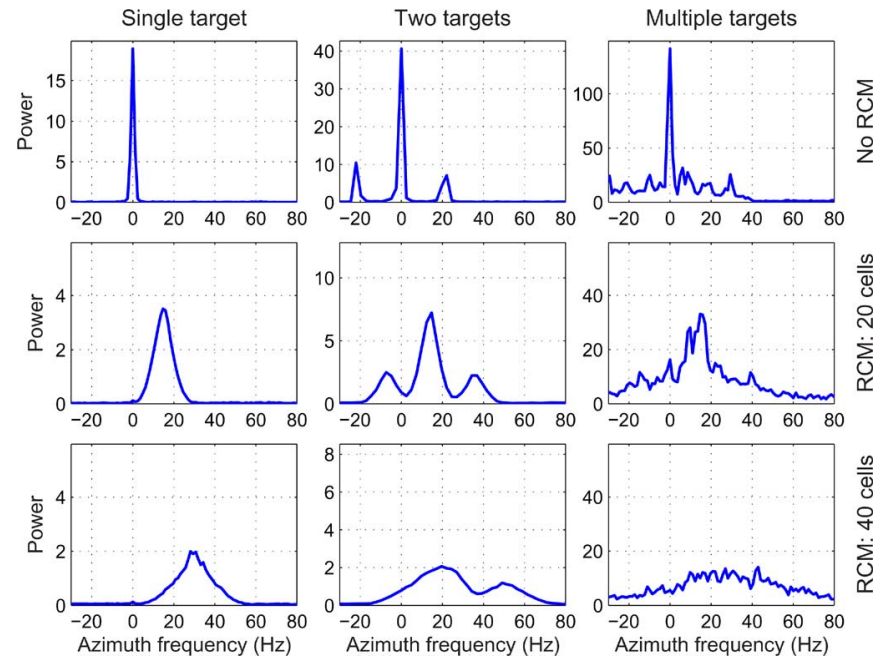

Fig. 5. Illustrating the effects of RCM on the beat signal resolution-single-, double-, and multiple-target cases.

is no RCM, the RCM has created two targets in each cell. This creates the cross-beating effect discussed in Section III-A, as long as the partial exposures overlap. Both of these effects reduce the sensitivity of the beat frequency estimation process. The cross-beating effect tends to get larger when a high density of targets is present, although the target overlap is reduced when the exposure within a cell is smaller.

\section{Illustration of the Effects of Cross Beating and RCM Combined}

Fig. 5 illustrates the effect of cross beating and RCM on the beat spectra using a discrete-target simulation. RADARSAT-1 FINE parameters are used, as in Fig. 3. Three cases of RCM are shown. In the first row, the Doppler centroid is set to zero; therefore, there is no linear RCM and only a small amount of quadratic RCM (about half a cell). The expected beat frequency is zero. In the second row, the Doppler centroid is set to 4.7 PRFs, giving the target(s) an RCM of 20 cells and an expected beat frequency of $15.56 \mathrm{~Hz}$. In the third row, the Doppler centroid is set to 9.4 PRFs, giving the target(s) an RCM of 40 cells and an expected beat frequency of $31.12 \mathrm{~Hz}$.

The first column shows the beat spectrum in the case of a single target. This allows the effect of limited target exposure time in one range cell to be isolated. When there is no RCM, the target is fully exposed in the range cell, and the resolution of the beat signal is the finest. However, when RCM is appreciable, the reduced exposure time in each range cell broadens the spectrum of the beat signal, and lowers the height of the peak. ${ }^{6}$

The second column shows the beat spectrum when there are two targets of the same strength in the same range cell, with a separation of 15 pulses, as in Fig. 3(b). In the first row, the $\mathrm{RCM}$ is zero; therefore, the exposure time is 700 pulses, and the target overlap is 685 cells. The long exposure time and overlap gives strong autobeat and cross-beat components, with a fine resolution.

\footnotetext{
${ }^{6}$ Note that the vertical scales of the plots in Fig. 5 have been adjusted for clarity.
} 
In the second row of Column 2, the exposure in each range cell has been reduced to about 45 cells by the RCM, and the target overlap reduced accordingly. The autobeat and the crossbeat signal strengths have been reduced, and the resolution of the peaks has been broadened by the reduced exposure. In the third row, the RCM has been increased to 40 cells, and the reduced exposure has further broadened the resolution to the point where the auto- and cross-beat signals are becoming mixed and the spectral peak is shifted.

The third column shows the results when there are multiple targets in each range cell. In this part of the simulation, 100 targets are randomly placed in a $30 \times 30$ sample square, with Rayleigh amplitude distribution and random phases. The cross beating is now much more complex and adds noiselike components to the spectrum.

In the first column, it is easy to see how the three values of Doppler centroid change the beat frequency from zero to 15.5 and $31 \mathrm{~Hz}$. With a single target in one range cell, the correct beat frequency is easily estimated. Even if there were other targets with much smaller magnitude present, the strong target would dominate the spectrum, and the beat frequency would likely be estimated correctly. However, the second and third columns show that when there are two or more targets of comparable magnitude present, the beat frequency can easily become obscured, possibly leading to Doppler ambiguity estimation errors. For example, in Row 3 of Column 2, the beat frequency has apparently been shifted from 31 to $20 \mathrm{~Hz}$.

\section{Discussion}

In summary, we have shown how multiple targets in a range cell causes cross beating, which introduces spurious signals into the spectrum of the beat signal. In addition, we have seen how RCM reduces the exposure time in one range cell, which broadens the resolution of the beat signal. Each of these effects can mask the appearance of the beat frequency from a dominant target and reduce the reliability of the Doppler ambiguity estimate.

\section{Estimation of the BeAt FREQUENCY}

We have seen that using only the linear term of (11) and under favorable cross-beating and RCM conditions, the beat signal is approximately a complex exponential, whose frequency must be estimated in order to find the absolute Doppler centroid frequency. For purposes of examining the frequency estimation problem, it is convenient to consider the beat signal (10) to be a complex exponential with additive noise

$$
\begin{aligned}
s_{\text {beat }}(n) \approx A(n) \exp \left\{j 2 \pi \frac{f_{\text {beat }}}{\mathrm{PRF}} n\right\}+z(n) & \\
n & =0,1,2, \ldots, N-1
\end{aligned}
$$

where $n$ is the azimuth time index, $A(n)$ is the azimuth envelope, and $z(n)$ is complex Gaussian noise with variance $\sigma^{2}$.

As the beat frequency (12) is proportional to the absolute Doppler centroid, the unambiguous centroid frequency can be estimated from the measurement of the beat frequency using

$$
f_{\eta_{c}}=-\frac{f_{0}}{\Delta f_{r}} f_{\text {beat }}
$$

The cross-beating and RCM effects described in Section III, as well as SNR found in some scenes, can make it difficult to estimate the beat frequency accurately [6]. However, we have found that the estimation can be improved by introducing better frequency estimators.

\section{A. Existing Estimator Using Fast FTs (FFTs)}

The existing MLBF algorithm uses FFTs to estimate the beat frequency [5]. The FFT forms a periodogram containing power estimates at discrete frequencies [7]. The estimation method is based on maximum likelihood estimation described by Rife and Boorstyn in [8]. The estimation equation is given by

$$
\begin{aligned}
& \hat{f}_{\text {beat }}=\frac{\mathrm{PRF}}{N_{\mathrm{FFT}}} \\
& \times \underset{0 \leq m<N_{\mathrm{FFT}}-1}{\arg \max }\left|\sum_{n=0}^{N-1} s_{\text {beat }}(n) \exp \left\{-j 2 \pi m n / N_{\mathrm{FFT}}\right\}\right|^{2}
\end{aligned}
$$

where $N_{\mathrm{FFT}}$ is the FFT length. The beat frequency is estimated by detecting the discrete frequency that corresponds to the biggest FFT coefficient. However, this method involves a quantization error because it only evaluates the energy at the discrete frequencies of the FFT. ${ }^{7}$ The maximum quantization error is

$$
\Delta f_{\eta_{c}}=\frac{f_{0}}{\Delta f_{r}} \frac{\Delta f_{\mathrm{FFT}}}{2}=\frac{1}{2} \frac{f_{0}}{\Delta f_{r}} \frac{\mathrm{PRF}}{N_{\mathrm{FFT}}} .
$$

In addition, the effect of RCM or burst mode operation broadens the beat signal bandwidth, further reducing the sensitivity of the method.

Since the existence of the quantization error and the RCM segmentation restrict the accuracy of the FFT estimator, more precise frequency estimators are studied to improve the beat frequency estimation. A literature search revealed that frequency estimators based on phase increments are the most promising for this Doppler ambiguity resolver (DAR) application. In this paper, we examine a number of estimators based on phase increments and evaluate them in the MLBF context.

\section{B. Basic Kay Estimator}

In 1989, Kay proposed a frequency estimator based on phase increments instead of the periodogram [9]. If it is assumed that the SNR is large, the additive noise model of (20) can be replaced by an approximate model using phase noise

$$
s_{\text {beat }}(n) \approx A(n) \exp \left\{j 2 \pi \frac{f_{\text {beat }}}{\mathrm{PRF}} n+u(n)\right\}
$$

\footnotetext{
${ }^{7}$ We found that using a "center of gravity" measure rather than the peak FFT coefficient gave a small improvement.
} 
where $u(n)$ is zero-mean white Gaussian phase noise with variance $\sigma^{2} /\left[2 A^{2}(n)\right]$. Then, the phase of the beat signal can be written as

$$
\arg \left\{s_{\text {beat }}(n)\right\}=2 \pi \frac{f_{\text {beat }}}{\text { PRF }} n+u(n)
$$

and the phase difference in the azimuth direction is given by

$$
\begin{aligned}
\Delta \arg \left\{s_{\text {beat }}(n)\right\} & =\arg \left\{s_{\text {beat }}(n+1)\right\}-\arg \left\{s_{\text {beat }}(n)\right\} \\
& =2 \pi \frac{f_{\text {beat }}}{\text { PRF }}+u(n+1)-u(n) .
\end{aligned}
$$

The differencing $[u(n+1)-u(n)]$ gives the noise a highpass characteristic; therefore, the problem is to estimate $f_{\text {beat }}$ from a signal with colored Gaussian noise. After some algebra, the optimal frequency estimate is found to be based on a weighted phase average

$$
\hat{f}_{\text {beat }}=\frac{\operatorname{PRF}}{2 \pi} \sum_{n=0}^{N-2} w(n) \Delta \arg \left\{s_{\text {beat }}(n)\right\}
$$

where $w(n)$ is a parabolic weighting function that allows the estimator to approach the Cramer-Rao bound [9].

The basic Kay estimator has the advantage that it is more computationally efficient than the optimal maximum likelihood estimator yet attains almost as good a performance. However, it has the disadvantage of having a relatively high SNR threshold. The SNR threshold refers to the SNR value, below which the estimation accuracy drops dramatically.

\section{ACCC and Other Frequency Estimators}

If the sum operation and the nonlinear arg operation are interchanged in the linear predictor (27), the estimate is modified a small amount. In this method, the parabolic weighting is replaced by the magnitudes of each signal increment $\left[\Delta s_{\text {beat }}(n)=s_{\text {beat }}(n+1)-s_{\text {beat }}(n)\right]$, and the estimate is proportional to the angle of the cross correlation coefficient (CCC) at lag one

$$
\hat{f}_{\text {beat }}=\frac{\mathrm{PRF}}{2 \pi} \arg \left\{\sum_{n=0}^{N-2}\left\{s_{\text {beat }}^{*}(n) s_{\text {beat }}(n+1)\right\}\right\} .
$$

Since this method uses the individual increment magnitudes as weighting, strong signals are emphasized, and the estimator can achieve better accuracy than Kay when the noise level is relatively high. In practice, the azimuth-direction CCC is averaged over range, and the procedure is referred to as the average CCC or ACCC method. It is worth noting that the use of the CCC was also proposed by Madsen in 1989 for baseband Doppler centroid estimation [10], for which it works very well [6].

Another method of improving Kay's estimator was proposed by Fitz in 1994 [11]. It uses the correlation at higher lags instead of only the correlation at lag one. Because the higher lag crosscorrelation coefficients provide more information of the average frequency of the noisy signal, this estimator can obtain better performance at moderate noise levels.
The Four Channel Filter Bank method was proposed by Fowler and Johnson in 1999 [12]. In this method, four simple filters are applied to overlapping frequency bands that cover the whole $(-\pi,+\pi]$ frequency range. The four channel filters work as bandpass filters, one of which is best at letting the beat signal pass through while removing the noise outside the pass band. Then, the signal in the filter channel with the highest energy is processed using Kay's estimator. The use of the bandpass filters to reduce the noise level gives this estimator better performance than the basic Kay estimator.

\section{Recommended Frequency Estimator-Iterative Linear Prediction (ILP)}

A more accurate estimator was proposed by Brown and Wang in 2002 [13], which is called the ILP method. This method uses iterative processing that successively reduces the filter bandwidth to improve the ACCC estimator.

In this algorithm, a coarse estimate of the frequency is first obtained by the ACCC estimator. The beat signal is bandshifted to baseband using the initial frequency estimate then lowpass filtered using a rectangular filter with coefficients $h_{i}=1$, where $i=0,1,2, \ldots, M-1$. The ACCC method is then used to estimate the frequency of the bandshifted, filtered signal. The low pass filter enhances the SNR by reducing the out-of-band noise, thereby improving the frequency estimate.

The estimate of the beat frequency is updated taking the bandshifting into account, and the next iteration starts by bandshifting the original beat signal by the refined frequency estimate. After each iteration, the bandwidth of the lowpass filter is reduced by doubling $M$. In this way, the SNR is enhanced by a narrower filter bandwidth, which improves the estimation accuracy at each iteration. The iterative procedure can be ended when there is no significant improvements detected or the bandwidth of the low pass filter is smaller than the bandwidth of the beat signal. We found that three iterations were sufficient, with $M=2,4$, and 8 , and that using a sinc filter instead of the simple averager obtains better results.

The iterative calculations are given by

Bandshifting : $\quad \tilde{s}_{\text {beat }}^{(k)}(n)=s_{\text {beat }}(n) \exp \left\{-j 2 \pi \frac{\hat{f}_{\text {beat }}^{(k-1)}}{\text { PRF }} n\right\}$

LPF and Subsample : $\nu^{(k)}(n)=\sum_{m=0}^{M_{k}-1} \tilde{s}_{\text {beat }}^{(k)}\left(n M_{k}+m\right)$
$n=0,1, \ldots, \frac{N}{M_{k}-1}$
ACCC $: \quad \Delta \hat{f}_{\text {beat }}^{(k)}=\frac{\text { PRF }}{2 \pi M_{k}} \arg \left\{\sum_{n=1}^{N / M_{k}-1} \nu^{(k)^{*}}(n-1) \nu^{(k)}(n)\right\}$

Update estimate : $\quad \hat{f}_{\text {beat }}^{(k)}=\left[\hat{f}_{\text {beat }}^{(k-1)}+\Delta \hat{f}_{\text {beat }}^{(k)}\right]_{\text {modulo } 2 \pi}$ 
where $\tilde{s}_{\text {beat }}^{(k)}$ is the bandshifted beat signal at iteration $k, \hat{f}_{\text {beat }}^{(0)}$ is the initial estimate of the basic ACCC estimator, $\nu^{(k)}(n)$ is the beat signal after lowpass filtering, $\Delta \hat{f}_{\text {beat }}^{(k)}$ is the estimated frequency error in the current iteration, and $\hat{f}_{\text {beat }}^{(k)}$ is the updated frequency estimate.

Experimental results described in Section VI show that the ILP estimator gives the best frequency estimation with a typical SAR scene. Therefore, we recommend that it be the estimator used for the MLBF Doppler ambiguity application.

\section{Applying RCM CORRECTION (RCMC) WITHIN MLBF}

In this section, we discuss the benefits of applying RCMC before the beat frequency is estimated. The benefits are illustrated using simulated data in Section V-A and using real SAR data in Section VI. An iterative scheme is needed because the correct RCMC depends on knowing the correct Doppler ambiguity - see Section V-B. Note that RCMC must be applied after range look extraction for reasons explained in the Appendix.

\section{A. Benefits of Applying RCMC}

When RCMC is applied with the correct ambiguity number, each target is confined to one range cell, and the beat signal arising from a target beating with itself (autobeating) has the largest magnitude and finest possible resolution. However, RCMC may bring other targets into the same range cell with a full exposure. The overlap with the existing target may be larger, giving rise to a stronger sharper cross-beat components. This effect depends on the distribution and strength of targets and may have the result of making the beat frequency estimation more difficult. However, in the simulations and real data examples we have tried, the RCMC effects have been beneficial, as shown below and in Section VI.

The simulation of Fig. 6 illustrates how RCMC can help to improve the beat frequency estimation. In the simulation, 100 targets are used, randomly placed within a $30 \times 30$ sample grid, giving an average of three targets in each range cell. Their amplitudes have a Rayleigh distribution, and their phases are random, as in Column 3 of Fig. 5. In each case, the beat spectrum has been averaged incoherently across range cells. The expected beat frequency is $20.42 \mathrm{~Hz}$.

Panel (a) shows the beat spectrum without applying RCMC. The RCM of 24 cells and the cross beating make the spectrum broad and noisy, and the peak frequency component is not at the expected place. But, after RCMC, a much cleaner narrower spectrum results in Panel (b) from an increase of the exposure time in each range cell and possibly from a reduction in the cross beating. The narrow peak allows an accurate estimation of the beat frequency. ${ }^{8}$

\section{B. Iterative Procedure of Applying RCMC}

RCMC can only be applied correctly if the Doppler ambiguity number is known, which is not available until after the

\footnotetext{
${ }^{8}$ Panel (c) is discussed in the Appendix.
}

(a)

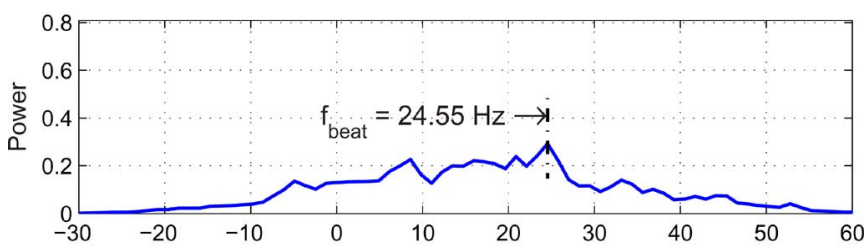

(b)

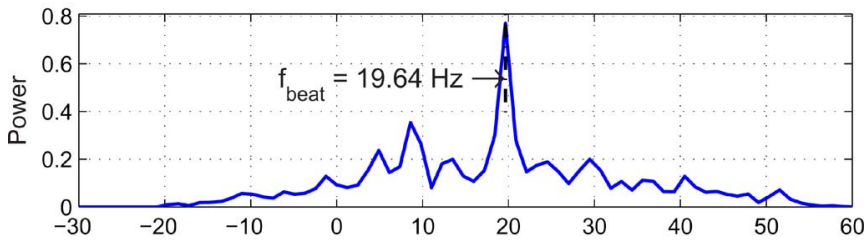

(c)

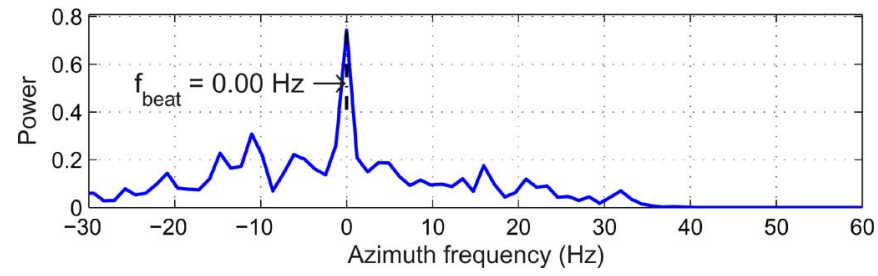

Fig. 6. Simulating the effects of RCMC on the beat signal resolution. (a) Multiple targets-before RCMC. (b) After applying RCMC after look extraction. (c) After applying RCMC before look extraction.

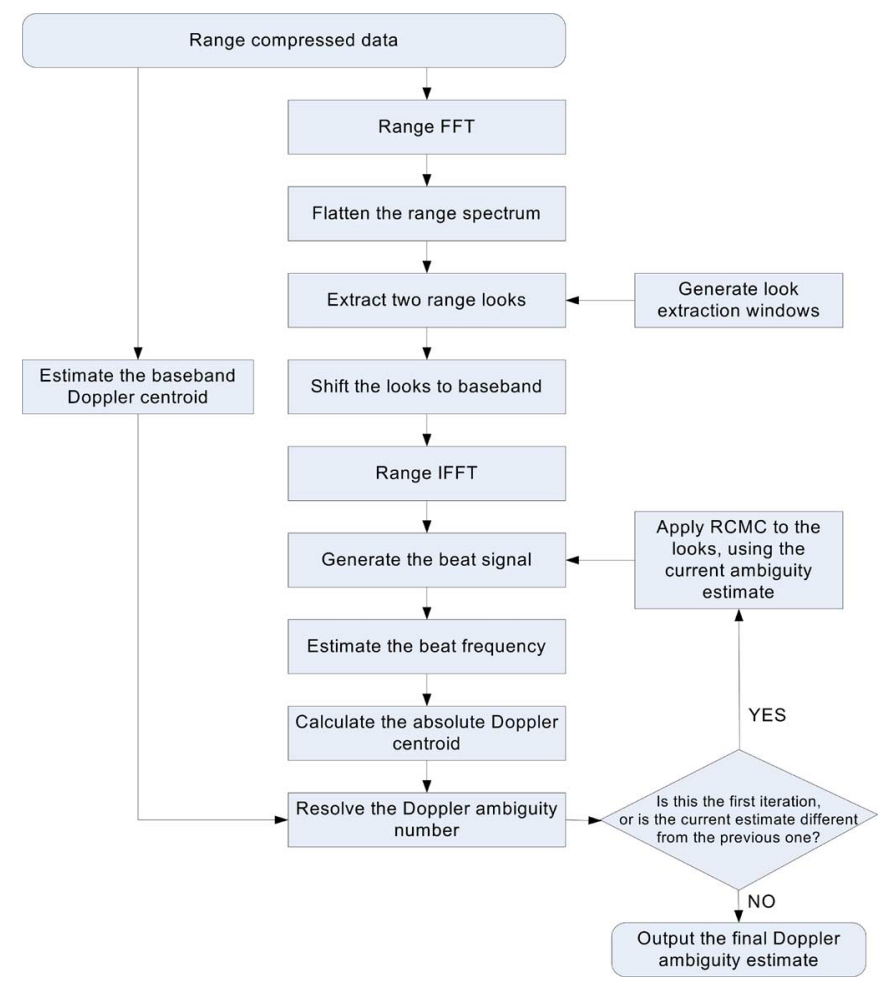

Fig. 7. Flowchart of the proposed RCMC/MLBF algorithm.

current estimation procedure is completed. Hence, in order to take advantage of the RCMC routine in the MLBF algorithm, an iterative procedure is proposed in Fig. 7.

In the first iteration, the conventional MLBF algorithm can be implemented without RCMC to obtain the initial estimate, or if available, an initial estimate of the Doppler centroid 
from geometry can be used. In subsequent iterations, RCMC is applied using the current Doppler ambiguity estimate. As the RCMC is refined, the bandwidth of the beat signal is reduced, and a lower bandwidth can be used to advantage in the ILP frequency estimator. The iterations continue until the Doppler ambiguity settles down to a stable value-we have found that usually only two iterations are required with real data (see Section VI). In fact, the frequency estimator works well even when RCMC is applied with a one ambiguity error. ${ }^{9}$

As the iterative algorithm requires applying the RCMC and the MLBF algorithm more than once, the computation time is a little more than the standard MLBF algorithm (the look extraction only needs be done once). We have found that the extra computation load is acceptable, as the iterative MLBF computation only occupies a small portion of the whole SAR processing time.

\section{Summary}

In summary, we have shown how RCMC can alleviate the effects of RCM and cross beating by increasing the resolution of the beat signal. We have shown that RCMC must be applied after the range look extraction (Appendix), and that an iterative scheme must be used because the correct ambiguity is needed to perform RCMC properly.

\section{EXPERIMENTAL RESUlTS With RADARSAT DATA}

The improved beat frequency estimators and the iterative RCMC method can improve the sensitivity of the MLBF algorithm, each in different ways. To test the performances of the two methods, we apply them to real SAR data of RADARSAT-1 using the FINE mode Vancouver scene of [6]. This scene is a representative test case, as it contains a wide variety of cultural and natural features, including areas of high and low contrast, and areas of high and low SNR. They included areas of fresh water, salt water, city, suburbia, farmland, deciduous forest, coniferous forest, and 1400-m mountains.

First, the RCMC benefit of Fig. 6 is further illustrated using specific areas of this scene. Then, in Section VI-B, the performance of the estimators is examined over the whole scene.

\section{A. Effect of RCMC on Specific Scene Types}

First, strong discrete targets consisting of the ships in Vancouver harbor are selected to provide a "best case" example of the benefits of RCMC, as the subscene contains only a few bright targets against a low-contrast background. ${ }^{10}$ The spectra of the beat signals are shown in Fig. 8 under conditions of no RCMC, RCMC applied with a one-ambiguity error, and RCMC applied with the correct ambiguity.

In Panel (a), cross-beating and RCM makes the spectrum asymmetrical, and the peak occurs at an incorrect frequency.

\footnotetext{
${ }^{9}$ In this method, as in other ambiguity resolvers, the baseband Doppler estimates are obtained first and unwrapped, so that only a single Doppler ambiguity number needs to be estimated for the whole scene.

${ }^{10}$ The range-compressed ships are shown in [6, Fig. 12.21].
}

(a)

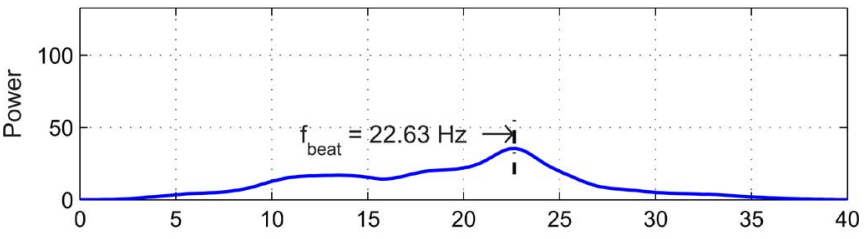

(b)

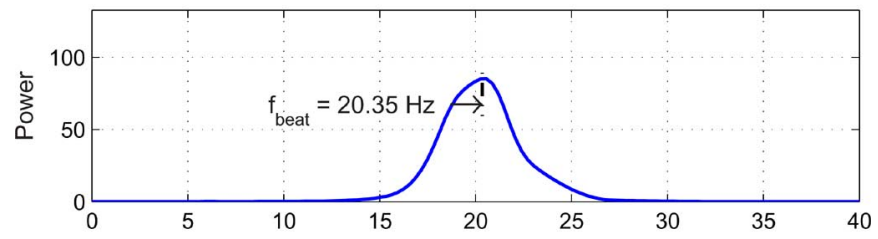

(c)

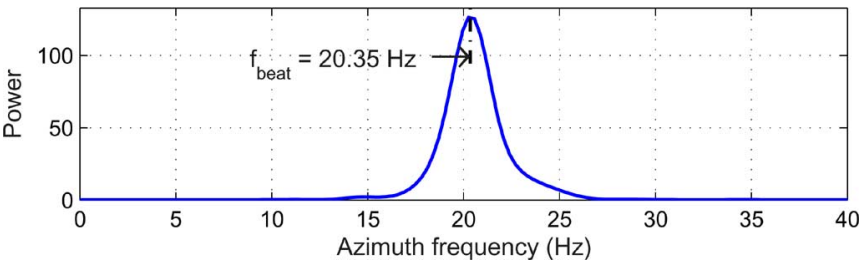

Fig. 8. Effect of RCMC on the beat signal spectrum in the "Vancouver ships" scene. (a) Beat spectrum without RCMC (ships scene). (b) Beat spectrum with RCMC (one-ambiguity error). (c) Beat spectrum with RCMC (no ambiguity error).

Then, RCMC is applied with iterations in which the ambiguity number is refined. Panels (b) and (c) illustrate how cross beating is reduced, and the resolution of the beat signal is sharpened as the iterations home in on the correct ambiguity number. While the sharpest spectral peak is obtained when RCMC is applied with the correct ambiguity number, Panel (b) shows that most of the sharpening effect is obtained even when there is a one-ambiguity error in applying RCMC with the C-band RADARSAT parameters.

In the "Vancouver ships" example, there are only a few strong targets in each range cell-a favorable case for the MLBF estimator. However, in lower contrast parts of the scene, the cross beating of a large number of similar-sized targets in each range cell can hide the peak in the spectrum. This effect is illustrated in Fig. 9, where a part of the Vancouver scene in the mountains is used. In this block of data, there is very low contrast, and no strong dominant targets are present. ${ }^{11}$

Panel (a) shows that the spectral peak is quite obscure compared to the high-contrast scene, and the wrong peak frequency is obtained when RCMC is not applied. Then, Panels (b) and (c) show how the spectrum is sharpened by the RCMC, and the correct beat frequency is obtained, even though the low scene contrast results in a spectral peak with a magnitude not much above the "noise" level. Again, when RCMC is applied with a one-ambiguity error, the peak is still visible, so the iterations of Section V-B should converge reliably.

\footnotetext{
${ }^{11}$ The mountainous part of the scene is taken from [6, Fig. 12.15, Row 18, Col. 12].
} 
(a)

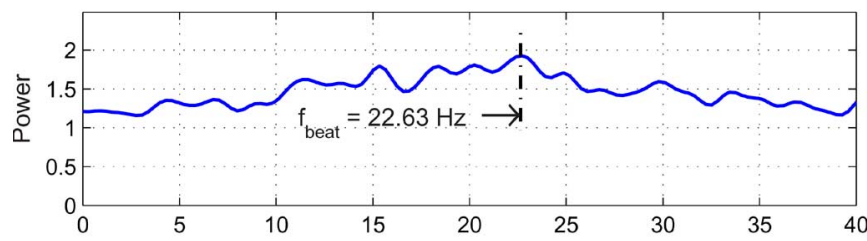

(b)

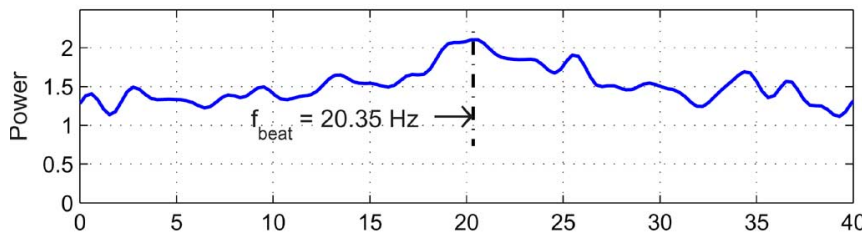

(c)

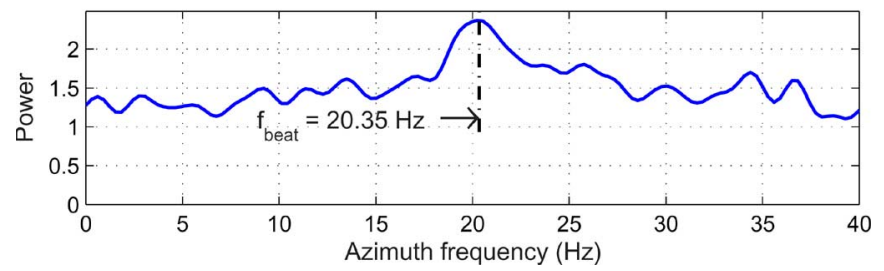

Fig. 9. Effect of RCMC on the beat signal spectrum with the Vancouver "mountains" scene. (a) Beat spectrum without RCMC (mountainous scene). (b) Beat spectrum with RCMC (one-ambiguity error). (c) Beat spectrum with RCMC (no ambiguity error).

\section{B. Experiments Over a Full SAR Scene}

In order to test the estimators over a variety of scene types, it is convenient to divide the Vancouver scene into small blocks, about $5 \mathrm{~km}$ across, so that the individual blocks represent a wide variety of estimation conditions. This approach is inspired by the "Spatial Diversity" concept [1], whose objective is to identify and isolate parts of the scene that are likely to lead to noisy or biased Doppler centroid estimates. In this paper, the range-compressed Vancouver data set is divided into 12 range $\times 19$ azimuth blocks. Each of the 228 blocks consist of 655 range cells and 1024 range lines (670 000 pixels per block).

The MLBF evaluation proceeds by applying the improved frequency estimators discussed in Section IV to obtain the beat frequency in each range cell, then the beat frequencies are averaged over range within the block. The absolute Doppler centroid is obtained from the beat frequency estimates using (21). Then, the Doppler ambiguity number for each block can be calculated using

$$
M_{\mathrm{amb}}=\operatorname{round}\left(\frac{f_{\eta_{c}}-f_{\eta_{c}}^{\prime}}{F_{a}}\right)
$$

where the baseband Doppler estimate $f_{\eta_{c}}^{\prime}$ is subtracted from the absolute estimate $f_{\eta_{c}}$, and the result is divided by the PRF (see $[6,12.20]$ ). The iterative RCMC method of Section V-B is also applied to estimate the Doppler ambiguity. After iterations with different RCMC slopes, this method yields one integer as the best Doppler ambiguity estimate for each block.

After applying the estimator to each of the blocks, the blocks with the possible bad estimates are removed using quality criteria. In order to compare the performances of all methods, the same quality criterion is applied (SNR $>-1 \mathrm{~dB})$, so that the same 21 blocks are removed in each experiment. Normally at this stage, a single Doppler ambiguity number is obtained for the whole scene using a "majority vote" or an average over the retained blocks. However, to assess the various estimators, we examine the statistics of the estimates of the individual blocks.

Table I shows the experimental results obtained using the improved frequency estimators and RCMC. First, the standard versions of the MLCC and MLBF estimators are given for comparison. Row 2 is the standard MLBF using the peak frequency observed at the FFT output. Row 3 also uses FFTs but finds the middle frequency using a center of gravity calculation. In Rows $4-8$, the improved frequency estimators described in Section IV are tested without RCMC. Finally, in Row 9, the iterative RCMC method is applied using the standard FFT frequency estimator.

The columns in Table I compare the statistics of the frequency estimates of each of the 207 nonrejected blocks of the scene. The mean value is shown in Column 3, indicating that the correct ambiguity number of -6 is obtained by all the tested estimators, as long as appropriate quality measures are used. However, it is interesting to examine the standard deviations, which are a suitable measure of the randomness of the estimates. It is shown that the methods based on the proposed frequency estimators using phase increments have a smaller standard deviation than the existing MLBF algorithm using FFTs, and that the ILP method gives the lowest standard deviation of the group. It has a standard deviation that is less than half of the existing MLBF algorithm and obtains the correct estimates in the highest percentage of blocks.

The iterative RCMC method shows an even better improvement. It has the highest success rate of all the estimators, as shown in the last column. Because the beat spectrum has a sharper peak and less noise after RCMC, the choice of frequency estimator is not so critical, and the simple FFT estimator works well in strip-map operations. ${ }^{12}$ It is interesting that using RCMC also gave substantial improvement to the "slope estimation" class of Doppler ambiguity estimators [14].

Fig. 10 shows the results of each nonrejected block in histogram form. While some blocks give large ambiguity errors, they are reasonably evenly distributed between plus and minus errors, so that a reliable ambiguity estimate is obtained when a consensus is taken over all the blocks of the scene. Note that the new estimators show significant improvement over both lowcontrast and high-contrast parts of the scene.

1) Discussion: It is well known that the MLBF algorithm works best with high-contrast scenes, while the MLCC algorithm works best with low-contrast scenes [5]. This fact is demonstrated in Fig. 11 using the blocks of the Vancouver scene, where the error of the algorithms is plotted against the block contrast. The contrast of each block is measured using

$$
C=E\left\{\left|P_{i, j}\right|^{2}\right\} / E\left\{\left|P_{i, j}\right|\right\}^{2}
$$

for each sample $P_{i, j}$ in the block.

\footnotetext{
${ }^{12}$ The standard deviation is higher than the ILP method because of the quantization of frequencies in the FFT.
} 
TABLE I

COMPARISON OF THE MLBF RESUlTS USING THE IMPROVED FREQUENCY ESTIMATORS AND RCMC

\begin{tabular}{|c|l|c|c|c|}
\hline & & $\begin{array}{c}\text { Mean } \\
(\mathrm{PRFs})\end{array}$ & $\begin{array}{c}\text { St. Dev. } \\
(\mathrm{PRFs})\end{array}$ & $\begin{array}{c}\text { Success rate } \\
(\%)\end{array}$ \\
\hline 1 & Standard MLCC & -5.99 & 1.36 & 41 \\
\hline 2 & Standard MLBF & -5.72 & 1.09 & 62 \\
\hline 3 & MLBF using Center of Gravity & -5.75 & 0.92 & 69 \\
\hline 4 & MLBF using Kay & -5.95 & 0.92 & 55 \\
\hline 5 & MLBF using ACCC & -6.02 & 0.90 & 57 \\
\hline 6 & MLBF using HLC & -5.94 & 0.75 & 63 \\
\hline 7 & MLBF using FCFB & -5.88 & 0.83 & 56 \\
\hline 8 & MLBF using ILP & -5.89 & 0.43 & 85 \\
\hline 9 & MLBF using Iterative RCMC & -5.92 & 0.73 & 92 \\
\hline
\end{tabular}
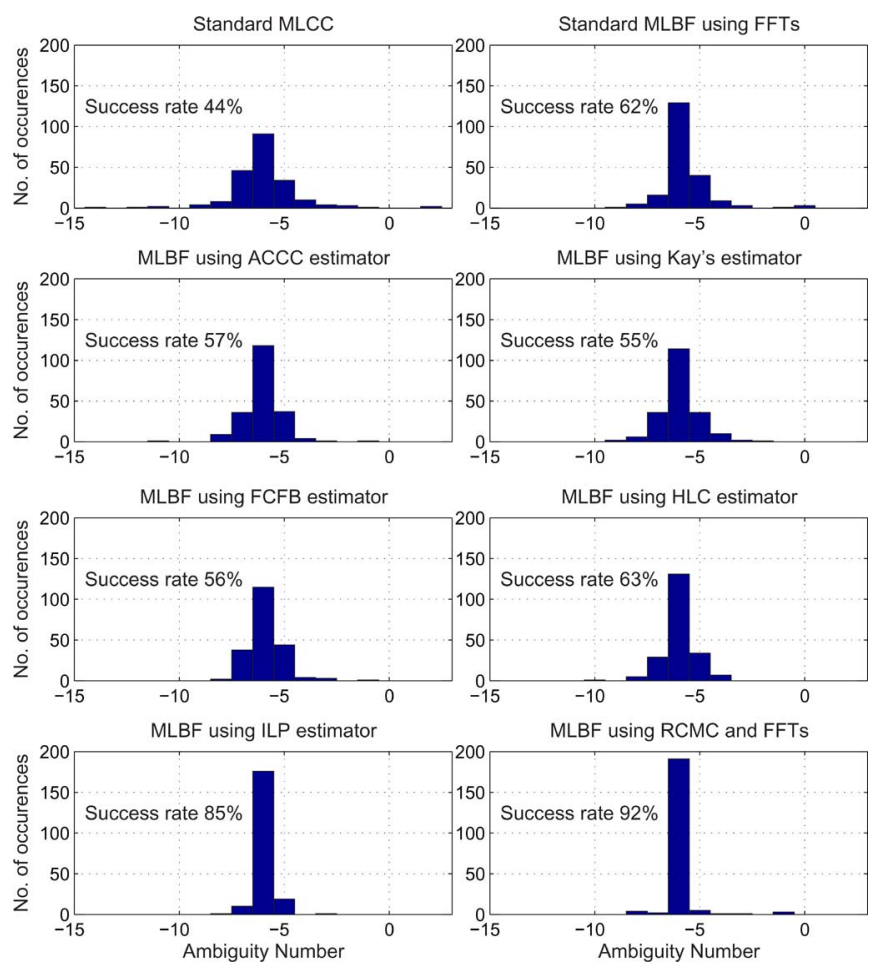

Fig. 10. Histogram of the Doppler ambiguity estimates by DARs of the 207 selected blocks.

In the MLCC algorithm, there is an increase in error as the contrast increases, but it is not too noticeable in the figure because there is a large concentration of low-error points in the vicinity of $C=1.5$. However, in the three MLBF cases, the errors clearly decrease with increased contrast.

\section{CONCLUSION}

The MLBF algorithm is one of the most reliable methods for Doppler ambiguity resolution and is widely used in practice. This paper explains the principle of the MLBF algorithm and proposes several ways to improve its sensitivity. In the basic algorithm, FFTs are used to estimate the frequency of the beat
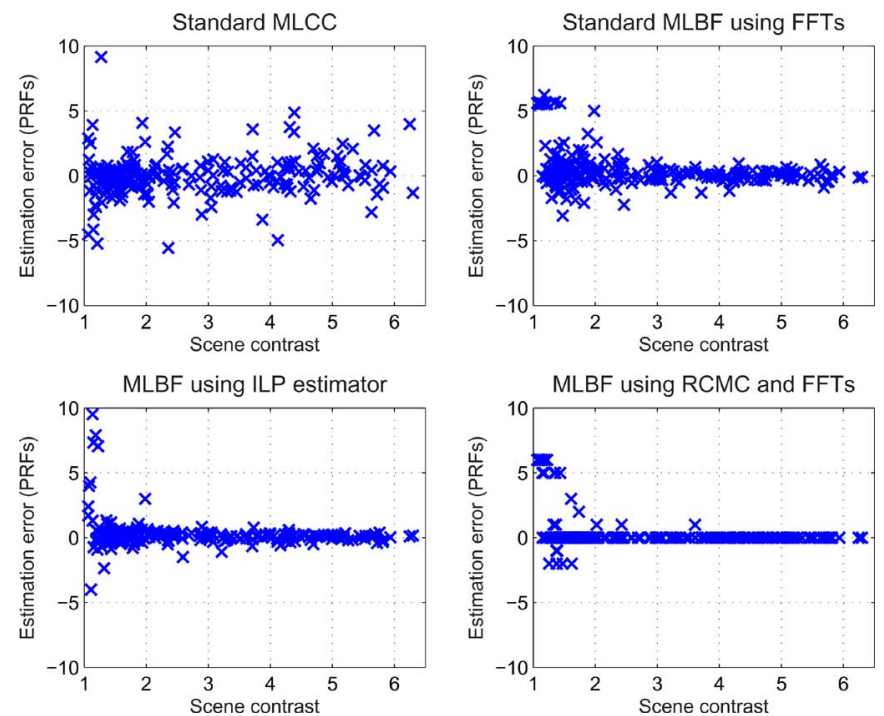

Fig. 11. Effect of image contrast on the performance of the estimators.

signal. However, the FFTs have limitations of resolution and quantization, especially when the signal is discontinuous in one range cell due to range cell migration or burst mode operation.

First, by examining the phase of the frequency-domain signal, we find that the azimuth phase variation induced by range migration plays an important role in the generation of the beat signal. The importance of having symmetrical range looks is highlighted. Then, two methods of improving the sensitivity of the algorithm are presented. One method uses more accurate frequency estimators based on the phase increments of the beat signal and noise rejection filters. A second method uses RCMC to straighten the target trajectories before the beat frequency estimator is applied. The RCMC has the effect of narrowing the bandwidth of the beat signal and reducing the effect of cross beating when more than one significant target is present in each range cell. An iterative scheme is used because the correct ambiguity number is needed to apply the RCMC.

Finally, experiments with RADARSAT-1 data are used to illustrate the improved estimation accuracy of the modified 
algorithms. Working with 228 contiguous blocks of 670000 pixels in the RADARSAT scene, quality criteria are used to reject areas of the scene that are likely to produce bad estimates. When the estimates of the 207 nonrejected blocks were examined, it was found that the percentage of blocks that yield the correct ambiguity number increased from $62 \%$ to $92 \%$ when the new MLBF algorithms were used. While the two algorithm modifications (RCMC and the ILP frequency estimator) can be combined, the majority of the estimation improvement can be obtained by using one of the modifications alone, as each method approaches the sensitivity problem in a different fashion. While the FFT-based frequency estimator works well with RCMCed data, it should not be used with ScanSAR data because of the fragmented azimuth phase histories.

\section{APPENDIX}

\section{Why RCMC Must Be APplied After LOOK EXTRACTION}

As illustrated in Section II-C, it is the slope of the phase ramp in the range-frequency domain that allows the two range looks to extract the relevant phase properties, and it is the variation along azimuth of this slope that gives rise to the distinctive beat frequency. Therefore, if RCMC is to be applied in the MLBF algorithm, one must be careful not to destroy this phase variation.

If we apply RCMC before range look extraction, the RCMC changes the target range from $R(\eta)$ to $R_{0}$ in all range lines in (1), where $R_{0}$ is the reference slant range for that target. This has the effect of removing the phase ramp along azimuth time after the range FFT, so that when the two range looks are extracted, there is no phase difference between the two looks.

To observe this, note that the azimuth phase ramp in the range-frequency domain in (1) has been changed from $-4 \pi f_{\tau} R(\eta) / c$ to $-4 \pi f_{\tau} R_{0} / c$ by the RCMC, so the range phase slope $-4 \pi R_{0} / c$ is no longer azimuth dependent. In other words, the range-compressed signal in the range-frequency domain of (1) has been changed to

$$
\begin{aligned}
& S_{\mathrm{RCMC}}\left(f_{\tau}, \eta\right)=A_{1}^{\prime} W_{r}\left(f_{\tau}\right) w_{a}\left(\eta-\eta_{c}\right) \\
& \times \exp \left\{-j \frac{4 \pi f_{0} R(\eta)}{c}\right\} \exp \left\{-j \frac{4 \pi f_{\tau} R_{0}}{c}\right\} .
\end{aligned}
$$

The second exponential factor shows that the slope of the phase ramp in $f_{\tau}$ is now the same in every range line. ${ }^{13}$ As a result, when RCMC is applied before look extraction, the beat signal can be expressed as

$$
\begin{aligned}
s_{\text {beat }}^{\prime}(\tau, \eta) & =s_{r_{1}}^{\prime *}(\tau, \eta) s_{r_{2}}^{\prime}(\tau, \eta) \\
& =\left|A_{1} w_{a}\left(\eta-\eta_{c}\right)\right|^{2} \exp \left\{-j 4 \pi \Delta f_{r} R_{0} / c\right\} .
\end{aligned}
$$

As the phase of the beat signal is constant in azimuth [compare (36) with (10)], the beat signal has zero frequency, and the information used to obtain a Doppler estimate has been lost.

\footnotetext{
${ }^{13}$ Note that the $R(\eta)$ dependence in the first exponential term still remains, because it was created by the demodulation process and is not affected by the RCMC. However, this phase term does not provide any information to the MLBF algorithm, as it is not dependent on range frequency.
}

\section{RCMC After Look Extraction}

On the other hand, if RCMC is applied after the baseband look extraction, the phases in the compressed pulses of two looks are not changed. When RCMC is applied to the signal (8) and (9), only the range envelope is changed from $p_{r}[\tau-$ $2 R(\eta) / c]$ to $p_{r}\left[\tau-2 R_{0} / c\right]$, but the phase term (the argument of the complex exponential) is not affected. Consequently, the azimuth phase difference of the two looks still maintains the correct dependence on the range migration. As a result, the correct Doppler estimate can be obtained from this phase variation.

These effects are illustrated in the Panels (b) and (c) of Fig. 6. In Panel (b), RCMC is applied after range look extraction, and the benefits of RCMC described in Section V-A are obtained. In Panel (c), however, RCMC has been applied before range look extraction, the beat frequency has been shifted to zero, and the distinctive beat frequency (12) has been lost.

\section{ACKNOWLEDGMENT}

The authors would like to thank F. Wong (MacDonald Dettwiler and Associates) for providing further explanation of the beat frequency concepts in Section II and the Appendix.

\section{REFERENCES}

[1] I. G. Cumming, "A spatially selective approach to Doppler estimation for frame-based satellite SAR processing," IEEE Trans. Geosci. Remote Sens., vol. 42, no. 6, pp. 1135-1148, Jun. 2004.

[2] I. G. Cumming, P. F. Kavanagh, and M. R. Ito, "Resolving the Doppler ambiguity for spaceborne synthetic aperture radar," in Proc. IGARSS, Zurich, Switzerland, Sep. 8-11, 1986, pp. 1639-1643.

[3] C. Y. Chang and J. C. Curlander, "Application of the multiple PRF technique to resolve Doppler centroid estimation ambiguity for spaceborne SAR," IEEE Trans. Geosci. Remote Sens., vol. 30, no. 5, pp. 941-949, Sep. 1992.

[4] R. Bamler and H. Runge, "PRF-ambiguity resolving by wavelength diversity," IEEE Trans. Geosci. Remote Sens., vol. 29, no. 6, pp. 997-1003, Nov. 1991.

[5] F. H. Wong and I. G. Cumming, "A combined SAR Doppler centroid estimation scheme based upon signal phase," IEEE Trans. Geosci. Remote Sens., vol. 34, no. 3, pp. 696-707, May 1996.

[6] I. G. Cumming and F. H. Wong, Digital Processing of Synthetic Aperture Radar Data-Algorithms and Implementation. Norwood, MA: Artech House, 2005.

[7] S. Kay, Modern Spectral Estimation: Theory and Application. Englewood Cliffs, NJ: Prentice-Hall, 1988.

[8] D. Rife and R. Boorstyn, "Single-tone parameter estimation from discrete-time observations," IEEE Trans. Inf. Theory, vol. IT-20, no. 5, pp. 591-598, Sep. 1974.

[9] S. Kay, "A fast and accurate single frequency estimator," IEEE Trans. Acoust., Speech, Signal Process., vol. 37, no. 12, pp. 1987-1990, Dec. 1989.

[10] S. N. Madsen, "Estimating the Doppler centroid of SAR data," IEEE Trans. Aerosp. Electron. Syst., vol. 25, no. 2, pp. 134-140, Mar. 1989.

[11] M. P. Fitz, "Further results in the fast estimation of a single frequency," IEEE Trans. Commun., vol. 42, no. 234, pp. 862-864, Feb.-Apr. 1994.

[12] M. Fowler and J. Johnson, "Extending the threshold and frequency range for phase-based frequency estimation," IEEE Trans. Signal Process., vol. 47, no. 10, pp. 2857-2863, Oct. 1999.

[13] T. Brown and M. M. Wang, "An iterative algorithm for single-frequency estimation," IEEE Trans. Signal Process., vol. 50, no. 11, pp. 2671-2682, Nov. 2002.

[14] I. G. Cumming and S. Li, "Improved slope estimation for SAR Doppler ambiguity resolution," IEEE Trans. Geosci. Remote Sens., vol. 44, no. 3, pp. 707-718, Mar. 2006. 


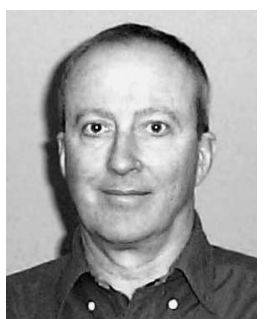

Ian G. Cumming (S'63-M'66) received the B.A.Sc. degree in engineering physics from the University of Toronto, Toronto, ON, Canada in 1961 and the $\mathrm{Ph} . \mathrm{D}$. degree in computing and automation from Imperial College, University of London, London, U.K., in 1968.

He joined MacDonald Dettwiler and Associates in 1977, and since that time, he has been developing synthetic aperture radar signal processing algorithms, including Doppler estimation and autofocus. He has been involved in the algorithm design of the digital SAR processors for SEASAT, SIR-B, ERS-1/2, J-ERS-1, and RADARSAT, as well as several airborne radar systems. He has also worked on systems for processing polarimetric and interferometric radar data, and the compression of radar data. In 1993, he joined the Department of Electrical and Computer Engineering, University of British Columbia (UBC), Vancouver, BC, Canada, where he held the MacDonald Dettwiler and Associates/Natural Sciences and Engineering Research Council Industrial Research Chair in radar remote sensing. The Radar Remote Sensing Laboratory supports a research staff of four engineers and students, working in the fields of SAR processing, SAR data encoding, satellite SAR two-pass interferometry, airborne along-track interferometry, airborne polarimetric radar classification, and SAR Doppler estimation. He retired from active teaching in 2005, but still supervises several graduate students at UBC, and is active in SAR consulting. In 2005, he published a book on SAR processing with coauthor Dr. F. Wong. The book describes the characteristics of the SAR received signal and the mainstream satellite SAR processing algorithms of range Doppler, chirp scaling, omega-K, and SPECAN.

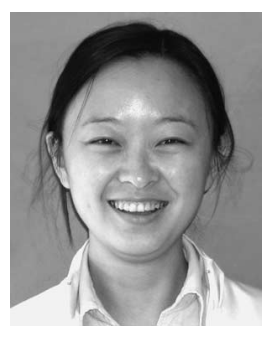

Shu Li received the B.E. degree from the Civil Aviation University of China, Tianjin, in 2000, the M.S. degree in electrical engineering from the Beijing Institute of Technology, Beijing, China, in 2003, and the M.A.Sc. degree from the University of British Columbia, Vancouver, BC, Canada, in 2005, in the field of satellite SAR Doppler centroid estimation. 